\title{
Small-Scale Fading Analysis of the Vehicular-to-Vehicular Channel inside Tunnels
}

\author{
Susana Loredo, ${ }^{1}$ Adrián del Castillo, ${ }^{2}$ Herman Fernández, ${ }^{3}$ Vicent M. Rodrigo-Peñarrocha, ${ }^{4}$ \\ Juan Reig, ${ }^{4}$ and Lorenzo Rubio ${ }^{4}$ \\ ${ }^{1}$ Electrical Engineering Department, University of Oviedo, C/Luis Ortiz Berrocal s/n, 33204 Gijón, Spain \\ ${ }^{2}$ Polytechnic School of Engineering, University of Oviedo, C/Luis Ortiz Berrocal s/n, 33204 Gijón, Spain \\ ${ }^{3}$ Escuela de Ingeniería Electrónica, Universidad Pedagógica y Tecnológica de Colombia, Sogamoso, Colombia \\ ${ }^{4}$ Electromagnetic Radiation Group (GRE), Universitat Politècnica de València, Camino de Vera s/n, 46022 Valencia, Spain
}

Correspondence should be addressed to Susana Loredo; loredosusana@uniovi.es

Received 20 July 2016; Revised 27 September 2016; Accepted 1 November 2016; Published 15 January 2017

Academic Editor: Oscar Esparza

Copyright (c) 2017 Susana Loredo et al. This is an open access article distributed under the Creative Commons Attribution License, which permits unrestricted use, distribution, and reproduction in any medium, provided the original work is properly cited.

\begin{abstract}
We present a small-scale fading analysis of the vehicular-to-vehicular (V2V) propagation channel at $5.9 \mathrm{GHz}$ when both the transmitter $(\mathrm{Tx})$ and the receiver $(\mathrm{Rx})$ vehicles are inside a tunnel and are driving in the same direction. This analysis is based on channel measurements carried out at different tunnels under real road traffic conditions. The Rice distribution has been adopted to fit the empirical cumulative distribution function (CDF). A comparison of the $K$ factor values inside and outside the tunnels shows differences in the small-scale fading behavior, with the $K$ values derived from the measurements being lower inside the tunnels. Since there are so far few published results for these confined environments, the results obtained can be useful for the deployment of $\mathrm{V} 2 \mathrm{~V}$ communication systems inside tunnels.
\end{abstract}

\section{Introduction}

Intelligent transportation systems (ITS) aim to improve safety and comfort of drivers and passengers through the integration of information and communication technologies both inside vehicles and in roadside infrastructure. The rapid development of wireless communication technologies has definitely contributed to the growing interest in vehicular communications by the automobile industry, governments, and academic institutions, enabling a wide range of potential applications [1-5].

Vehicular communications are usually classified into three categories: vehicular-to-vehicular (V2V) [6], vehicularto-infrastructure (V2I) [7], and vehicular-to-roadside (V2R) [8], which may interact together to form vehicular ad hoc networks (VANETs). The more challenging nature of V2V communications, due to the mobility of the two communication ends, may be the reason why they have attracted much more attention from the point of view of research and development. One of the key features for efficient implementation of such vehicular communication systems is to have a good characterization and therefore a deep understanding of the $\mathrm{V} 2 \mathrm{~V}$ propagation channel. Consequently, an important effort has been made in recent years in deepening the knowledge of $\mathrm{V} 2 \mathrm{~V}$ channel, which includes both measurement campaigns and channel modeling [6,9-13]. In order to have a full channel characterization, different environments (rural, suburban, urban, and highway) with different traffic conditions have been considered. Nevertheless, other particular environments are also of interest, such as parking garages [14] or tunnels $[15,16]$, which have not received so much attention so far.

Tunnels are confined environments, where the propagation conditions differ from other environments [17]. Under the ITS concept, the understanding of the V2V channel characteristics in these peculiar environments is essential for successful deployment of future safety applications based on wireless communication technologies. In this sense, we present a statistical analysis of the small-scale fading of the $\mathrm{V} 2 \mathrm{~V}$ channel inside tunnels from experimental channel measurements. This work has been developed in the framework of a more extensive measurement campaign that also 


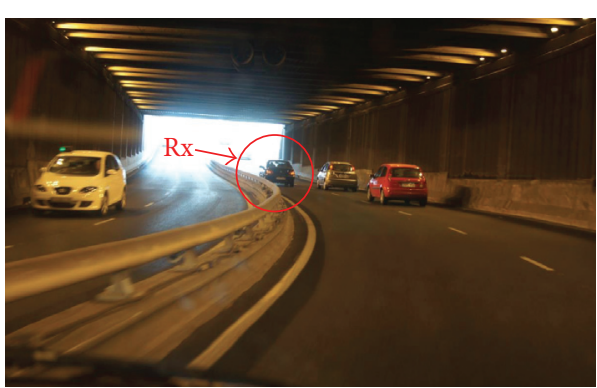

(a)

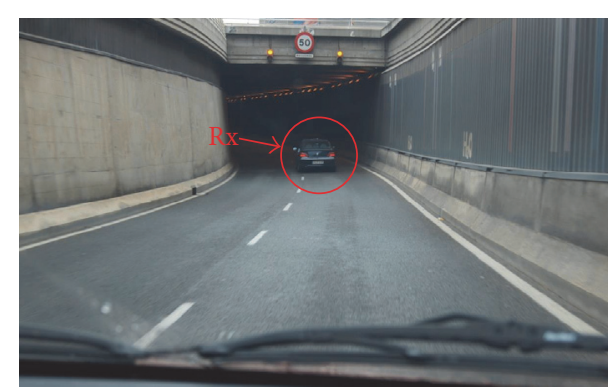

(b)

FIgure 1: (a) Two-way, two-lane tunnel in Track 1. (b) One-way, two-lane tunnel in Track 3. The pictures were taken from the Tx vehicle.

included channel measurements outside tunnels, so that a comparison inside/outside can be made. Measurements were performed at the $5.9 \mathrm{GHz}$ dedicated short-range communications (DSRC) frequency band under real road traffic conditions.

The remainder of the paper is organized as follows. Section 2 introduces the measurement setup and outlines the environments where the measurements have been taken. The small-scale fading is analyzed in Section 3. Finally, some concluding remarks are presented in Section 4.

\section{Channel Measurements}

2.1. Measurement Setup. The measurements used in this study are part of a more extensive V2V measurement campaign carried out around the city of Valencia (Spain). Narrowband propagation channel measurements were performed under different propagation and road traffic conditions [12]. At the transmitter side (Tx vehicle), the HP83623A signal generator (SG) was used to transmit an unmodulated continuous wave at $5.9 \mathrm{GHz}$. A high power amplifier (HPA) was used to obtain equivalent isotropic radiated power (EIRP) of $+23.8 \mathrm{dBm}$. At the receiver side ( $\mathrm{Rx}$ vehicle), the ZVA24 vector network analyzer (VNA) of R\&S was used to measure the received power level directly through the $b_{2}$ parameter (the $\mathrm{Rx}$ antenna was connected to Port 2 of the VNA). The VNA was configured to measure the $b_{2}$ parameter continuously using traces of 5000 test points. As a compromise between the thermal noise level and the measurement acquisition time, an intermediate frequency (IF) filter bandwidth equal to $100 \mathrm{kHz}$ was used in the VNA, resulting in a noise level of around $-70 \mathrm{dBm}$ and a sweep time per trace of about $220 \mathrm{~ms}$. Notice that the sweep time per trace corresponds to a sampling time (acquisition time of a test point) of about $44 \mu \mathrm{s}$. It is also worth noting that the bandwidth of the IF filter is higher than the maximum Doppler spectrum in vehicular environments, where the maximum Doppler frequency is in the order of few $\mathrm{kHz}$. For example, there is a maximum Doppler frequency of about $1 \mathrm{kHz}$ for a relative speed of $55 \mathrm{~m} / \mathrm{s}(198 \mathrm{~km} / \mathrm{h})$ between vehicles [18].

Two medium power amplifiers (MPAs) and low-loss cables were also used at the $\mathrm{Rx}$ side. Both transmitting and receiving antennas were omnidirectional monopoles
TABLE 1: Characteristics of the measured tracks.

\begin{tabular}{|c|c|c|c|c|}
\hline & $\begin{array}{l}\text { Total track } \\
\text { time }(\mathrm{s})\end{array}$ & $\begin{array}{c}\text { Tunnel } \\
\text { length (m) }\end{array}$ & $\begin{array}{l}\text { In-tunnel } \\
\text { time (s) }\end{array}$ & $\begin{array}{c}\text { Tunnel } \\
\text { characteristics }\end{array}$ \\
\hline Track 1 & 300 & 188 & 17 & $\begin{array}{l}\text { Two-way, } \\
\text { two-lane }\end{array}$ \\
\hline Track 2 & 180 & 515 & 47 & $\begin{array}{l}\text { Two-way, } \\
\text { two-lane }\end{array}$ \\
\hline Track 3 & 600 & 720 & 58 & $\begin{array}{l}\text { One-way, } \\
\text { two-lane }\end{array}$ \\
\hline Track 4 & 139 & 316 & 26 & $\begin{array}{l}\text { One-way, } \\
\text { two-lane }\end{array}$ \\
\hline
\end{tabular}

vertically polarized and roof-mounted in the center of the vehicles through a magnetic base. Two laptops, inertial GPS receivers, and a video camera completed the measurement system. More details about the measurement setup can be found in $[12,18]$.

2.2. Measurement Environments. The common feature of all the measurements used in this study is that there is always a tunnel section along the different tracks followed by the $\mathrm{Tx}$ and $\mathrm{Rx}$ vehicles. Data taken in four different tracks of the vehicles, in urban environment, specifically in the city of Valencia, are available. They will be called Track 1, Track 2, Track 3, and Track 4. Table 1 summarizes some characteristics of the different tracks. There are both double and one-way tunnels, as shown in Figure 1. Both vehicles were driven in the same direction (convoy) at a similar speed, close to $50 \mathrm{~km} / \mathrm{h}$ $(14 \mathrm{~m} / \mathrm{s})$, that is, the maximum allowable speed in the urban area.

As an example of a measurement record, Figure 2 shows the received power while the vehicles go over Track 3. It can be seen how the small-scale fading behavior outside and inside the tunnel is different. From the results shown in Figure 2, the fading depth of the received signal is lower outside (in blue color) than inside (in green color) the tunnel. Figure 3 shows the Tx and Rx speed (a) and Tx-Rx separation distance (b) for the measurement record of Figure 2.

\section{Small-Scale Fading Analysis}

In order to analyze the small-scale fading, the local mean received power was estimated for each track using a sliding 


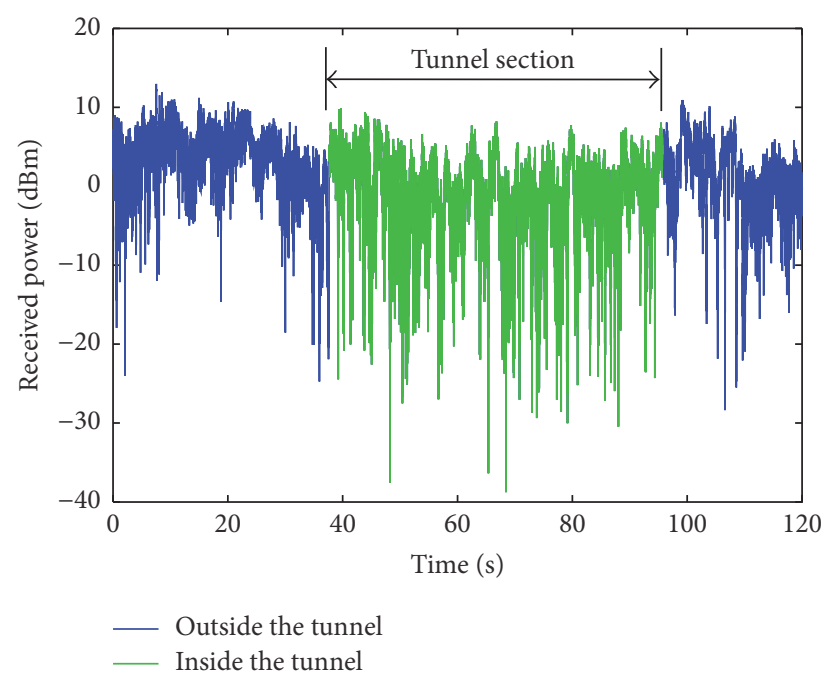

FIGURE 2: Received power outside and inside the tunnel for a measurement record in Track 3.

average window. Raw data was then normalized to the local mean power, and tunnel sections were identified in order to analyze separately the fading inside and outside the tunnels.

Narrowband fading statistics in fixed-to-mobile (F2M) channels are commonly assumed to be Rayleigh for nonline-of-sight (NLOS) links and Rice for line-of-sight (LOS) links, although other distributions such as Nakagami-m and Weibull have also been successfully used to fit the fading behavior. All of them have also been considered to characterize the small-scale fading distribution in $\mathrm{V} 2 \mathrm{~V}$ communication channels [19]. More specifically, the Rice and Nakagami- $m$ distributions have been used in $[9,10]$. The Nakagami- $m$ distribution is generally assumed to be a more general model, which would approximate Rice distribution when $m>1$, Rayleigh distribution for $m=1$, and fading more severe than Rayleigh when $m<1$ [20]. Thus, in [9], due to the variety of driving scenarios analyzed, the Nakagami$m$ distribution was chosen. The results show that the fading follows a Rice distribution for short distances between Tx and $\mathrm{Rx}$, decreasing the value of $m$ as the distance increases and reaching values lower than 1 for the largest distances. The authors in [9] attribute this last result to an intermittent loss of sight due, for example, to a vehicle turning a corner. In [10], both distributions were used to fit the measured data, finding that the Rice distribution yielded the smallest error due to the large number of LOS situations measured.

For the measurements under study, Tx-Rx distances were generally short and LOS did exist in most parts of the vehicles routes, particularly inside tunnels, where LOS could only be obstructed by a higher vehicle driving between Tx and Rx. The Rice distribution was initially chosen to perform the analysis of the measured data.

Tunnel sections were firstly analyzed. Since the fading behavior exhibits a high variability along a given track (even inside the tunnels), each fading record was divided into temporal bins within which the fading behavior could be considered uniform. Initially temporal bins of approximately
2.1 seconds were considered, and the Rice $K$ factor was estimated inside each. The value of $K$ was calculated from the received signal using the expression given in [21]

$$
\begin{aligned}
\frac{E\{r\}}{\sqrt{E\left\{r^{2}\right\}}}= & \sqrt{\frac{\pi}{4(K+1)}} \exp \left(-\frac{K}{2}\right) \\
& \times\left[(K+1) I_{0}\left(\frac{K}{2}\right)+K I_{1}\left(\frac{K}{2}\right)\right],
\end{aligned}
$$

where $I_{n}(\cdot)$ is the modified Bessel function of the first kind and $n$th order. $E\{r\}$ and $E\left\{r^{2}\right\}$ are, respectively, the mean and mean squared value of the Rice distribution, which are estimated from the measured samples as

$$
\begin{gathered}
E\{r\}=\frac{1}{N} \sum_{i=1}^{N} r_{i}, \\
E\left\{r^{2}\right\}=\frac{1}{N} \sum_{i=1}^{N} r_{i}^{2},
\end{gathered}
$$

with $r_{i}$ being the square root of the measured power samples. Once the ratio $E\{r\} / E\left\{r^{2}\right\}$ is known, the value of $K$ can be easily estimated, which is graphically shown in [21, Fig. 4].

The Kolmogorov-Smirnov (K-S) test [22, 23] was used to evaluate the goodness of fit of the estimated Rice distribution to the empirical data. The statistic $D$ used in the K-S test to compare the hypothesized and empirical cumulative distribution functions (CDFs) is the maximum value of the absolute difference between both curves. The Rice hypothesis will be accepted if the value of $D$ is not higher than a threshold value $d_{N, \alpha}$, which depends on both the number of samples, $N$, and the significance level, $\alpha$. For high values of $N$ (typically $N>35$ ),

$$
d_{N, \alpha}= \begin{cases}\frac{1.63}{\sqrt{N}}, & \alpha=0.01 \\ \frac{1.36}{\sqrt{N}}, & \alpha=0.05 \\ \frac{1.22}{\sqrt{N}}, & \alpha=0.1 .\end{cases}
$$

Figure 4(a) shows the small-scale fading for the tunnel section of Track 1, and Table 2 summarizes the values estimated for $K$ and the corresponding values found for $D$. For a significance level of $1 \%$, two values of $D$ are above the threshold $\left(d_{N, \alpha=1 \%}=0.0729\right)$ given by (1); that is, the percentage of fulfillment of the K-S test for this example would be $75 \%$.

Figure 4(b) shows the values estimated for $K$ when a halfsize window, that is, about one-second bin, is considered, proving that inside temporal bins of Table 2 fading may still experience a nonnegligible variation, and a shorter temporal bin could be necessary in order to ensure greater fading stability. A temporal bin of $1.05 \mathrm{~s}$ is coherent with the stationary times found in [15]. It must be noted that in our measurements the scattering environment is expected to change more slowly due to the lower speed of the vehicles; therefore, stationary times should increase. 


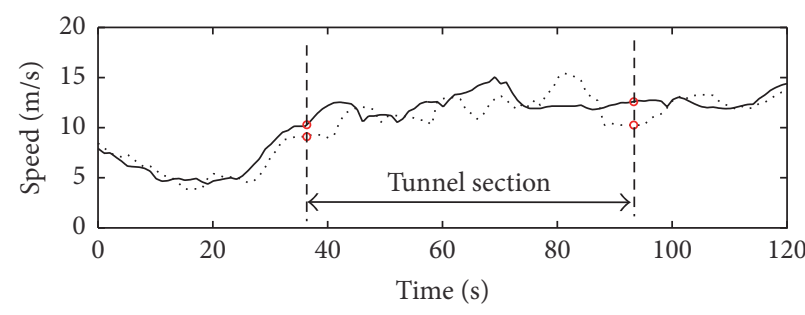

… Tx

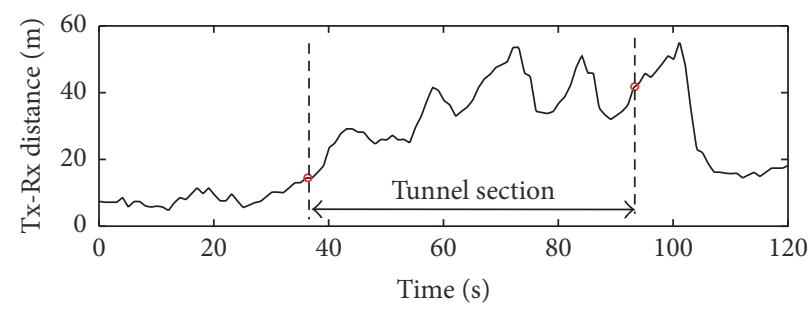

(a)

(b)

Figure 3: Tx and Rx speed (a) and Tx-Rx separation distance (b) for a measurement record in Track 3.

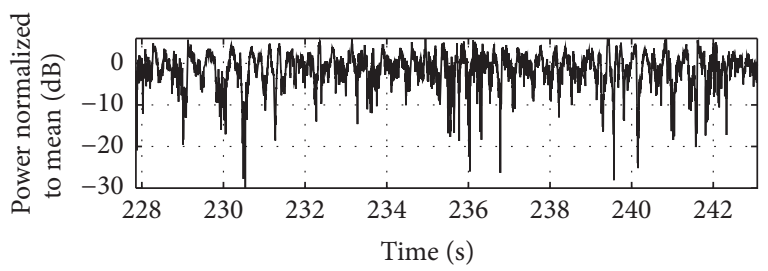

(a)

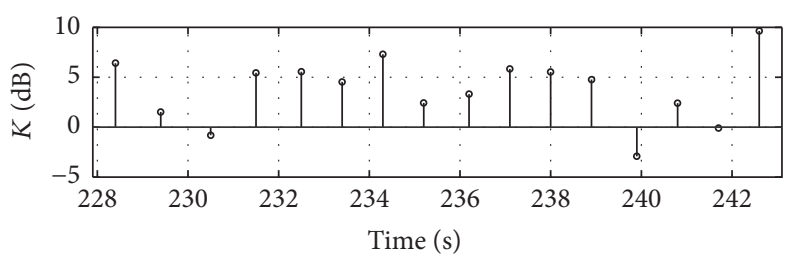

(b)

FIGURE 4: Tunnel of Track 1: (a) received power without shadowing component and (b) variation of $K$ values when estimated in 1.05-second temporal windows.

TABLE 2: Estimated values of $K$ parameter and $D$ statistic for the temporal bins of tunnel section in Track 1 .

\begin{tabular}{lcc}
\hline Temporal bin $(\mathrm{s})$ & $K(\mathrm{~dB})$ & $D$ \\
\hline$[227.9-230.0]$ & 4.0 & 0.0893 \\
{$[230.0-232.1]$} & 2.8 & 0.0357 \\
{$[232.1-234.2]$} & 4.9 & 0.1017 \\
{$[234.2-236.3]$} & 4.8 & 0.0239 \\
{$[236.3-238.4]$} & 4.5 & 0.0585 \\
{$[238.4-240.5]$} & 5.1 & 0.0664 \\
{$[240.5-242.6]$} & 0.0 & 0.0236 \\
{$[242.6-243.1]$} & 4.5 & 0.0303 \\
\hline
\end{tabular}

When diminishing the temporal bin, the percentage of fulfillment of the K-S test for the same tunnel section is $81.25 \%$ both for $1 \%$ and for $5 \%$ significance levels. In case that the vehicles speed changes significantly along the route, a variable size should be defined for the temporal bins taking into account the instantaneous vehicles speed in order to maintain a given spatial resolution. However, in these measurements, the speed was always fairly uniform, as can be seen in the example of Figure 3(a), and so the spatial resolution is approximately the same at the different bins.

In order to facilitate the comparison among tunnels and also compare fading inside and outside the tunnels, the mean value of $K$ parameter for the whole analyzed fading curve will be used. Figure 5 compares the CDFs of fading inside and outside the tunnel of Track 1, showing the existence of deeper fades inside the tunnel and consequently stronger multipath components (MPCs). The same is found for the

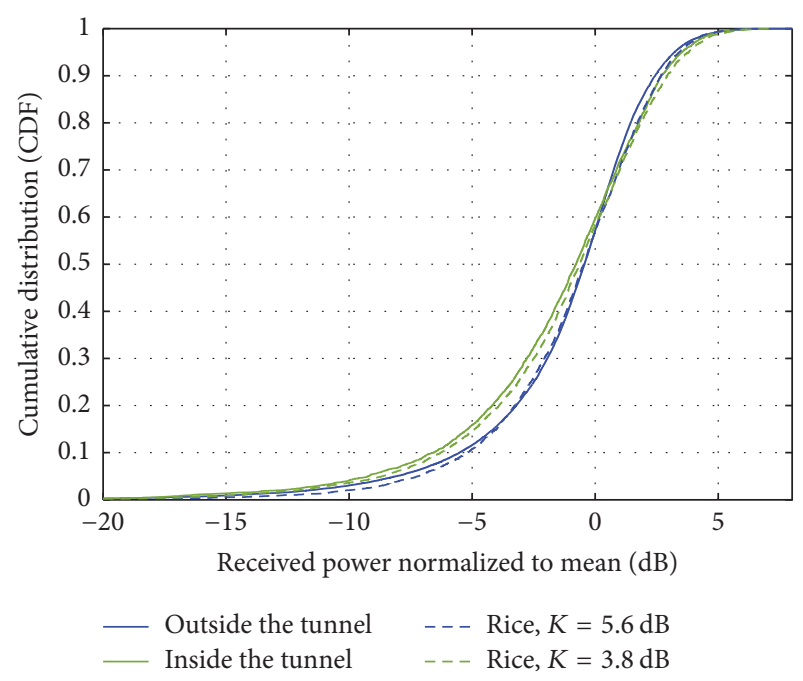

FIGURE 5: Comparison between CDFs of fading inside and outside the tunnel of Track 1 .

other tracks, with the mean value of $K$ being always higher outside than inside the tunnels, as can be seen in Table 3, which summarizes the mean values of $K$ for the different tracks, both inside and outside the tunnels. This result is consistent with that published in [15] from wideband channel measurements, where the root-mean-square (rms) delay and Doppler spreads are found to be higher inside the tunnel than in "open-air" situations, as a consequence of the observed strong MPCs caused by reflections from tunnel walls, ceiling, and other structures inside the tunnel. Furthermore, the 
TABLE 3: Mean values of $K(\mathrm{~dB})$ inside and outside the tunnels.

\begin{tabular}{lcc}
\hline & Inside the tunnel & Outside the tunnel \\
\hline Track 1 & 3.8 & 5.6 \\
Track 2 & 3.0 & 4.9 \\
Track 3 & 5.2 & 7.4 \\
Track 4 & 6.4 & 8.6 \\
\hline
\end{tabular}

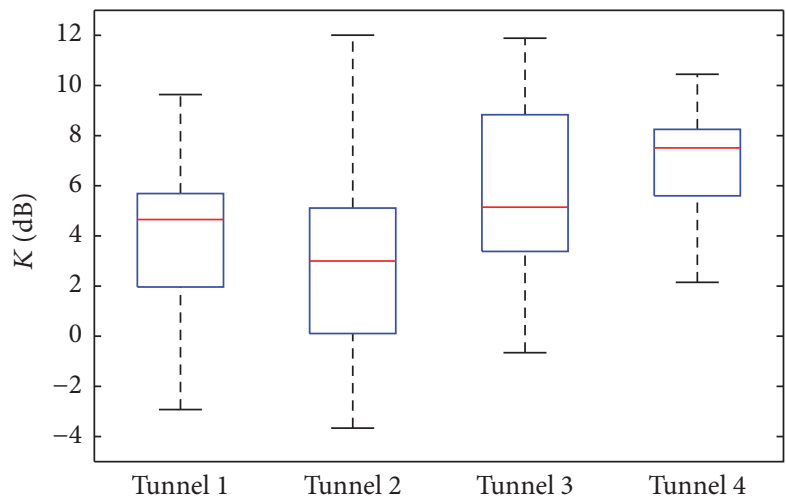

Figure 6: Boxplot of the $K$ factor inside the tunnels.

TABLE 4: 25th, 50th, and 75th percentiles of $K(\mathrm{~dB})$ factor values inside the tunnels.

\begin{tabular}{lcccc}
\hline Percentile & Tunnel 1 & Tunnel 2 & Tunnel 3 & Tunnel 4 \\
\hline 25th & 1.9 & 0.2 & 3.3 & 5.6 \\
50th & 4.6 & 2.9 & 5.1 & 7.4 \\
75th & 5.7 & 5.1 & 8.8 & 8.2 \\
\hline
\end{tabular}

values for $K$ outside the tunnels agree with those estimated in [19] from different routes in urban environments. There, it was found that the mean of the Rice $K$ parameter ranged from 4.2 to $8.8 \mathrm{~dB}$.

Also in Table 3, one can observe that the mean values of $K$ for the tunnels in Track 1 and Track 2 are similar and lower than the values in the tunnels of Track 3 and Track 4. This can be attributed to the different topology of the tunnels analyzed. In the first ones (two-way, two-lane), the number of vehicles acting as potential scatterers theoretically doubles in relation to the tunnels of Track 3 and Track 4 (one-way, two-lane), leading to higher multipath and lower values of $K$.

The variability of $K$ factor inside the tunnels was also studied and it is illustrated in Figure 6 using a boxplot. The red line inside the box is the median value (50th percentile), whereas the lower and upper edges of the blue box correspond to the 25 th and the 75 th percentiles. These values are summarized in Table 4 . The variation of $K$ in terms of standard deviation relative to the mean (Table 3 ) has also been calculated, obtaining 3.2, 3.7, 3.2, and $2.8 \mathrm{~dB}$ for tunnels $1,2,3$, and 4 , respectively.

In order to quantify the error of this approximation, which attempts to characterize the complete fading curve by a unique value of $K$, the fitting error is calculated as the absolute difference between the fitted analytical CDF and the data empirical CDF. This error is shown in Figure 7 for the

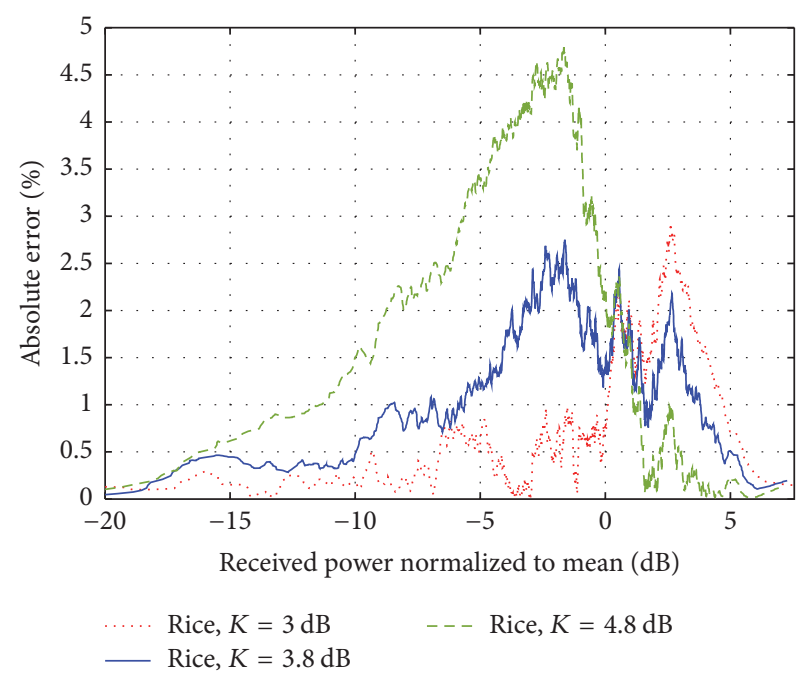

FIGURE 7: Tunnel of Track 1: error of the fitting by Rice distribution.

tunnel of Track $1(K=3.8 \mathrm{~dB})$. The magnitude of the error is lower than that reported in [9]. Furthermore, the error diminishes if the lower and upper regions of the CDF are fitted for different Rice distributions: a $K$ value slightly lower than the estimated one, for example, $K=3 \mathrm{~dB}$, fits better the lower part of the curve, that is, the deeper fades, whereas function values higher than the mean $(0 \mathrm{~dB})$ are better fitted by a slightly higher $K$ value, for example, $K=4.8 \mathrm{~dB}$.

\section{Conclusion}

A small-scale fading analysis of the V2V channel inside tunnels has been presented. The results have been derived from narrowband channel measurements collected in four different tunnels under real road traffic conditions. The Rice distribution has been proven to provide a reasonable fit to the measured small-scale fading when the size of the temporal bin is properly chosen.

The fading was also characterized globally through a mean value of $K$ for each whole fading record, in order to have a parameter to facilitate the comparison between tunnels and also inside/outside tunnels. For the different routes followed by the vehicles, this mean $K$ factor is always higher outside than inside the tunnel, indicating a stronger multipath effect inside the tunnels. Furthermore, inside the tunnels, fading has turned out to be more severe in two-way tunnels with higher number of lanes, due to the existence of a higher number of scatterers. This result suggests a possible classification of the tunnels attending to these criteria. In this sense, more measurement campaigns developed in tunnels of different cities and fading modeling activities are necessary to have a better understanding of the small-scale fading behavior in these confined environments.

For this global characterization, it has also been shown that the fitting error diminishes when two different values of $K$ are used for the lower and higher parts of the CDF. This may be explained by taking into account the changing nature 
of the fading along a given route, which makes adjusting its behavior by a single statistical parameter not possible.

\section{Competing Interests}

The authors declare that there are no competing interests regarding the publication of this paper.

\section{Acknowledgments}

The authors want to thank J. A. Campuzano, D. Balaguer, and L. Moragón for their support during the measurement campaign, as well as B. Bernardo-Clemente and A. VilaJiménez for their support and assistance in the laboratory activities. This work has been funded in part by Programa Estatal de Fomento de la Investigación Científica y Técnica de Excelencia del Ministerio de Economía y Competitividad, Spain, TEC2013-47360-C3-3-P, and Departamento Administrativo de Ciencia, Tecnología e Innovación COLCIENCIAS en Colombia.

\section{References}

[1] S. Tsugawa, "Inter-vehicle communications and their applications to intelligent vehicles: an overview," in Proceedings of the IEEE Intelligent Vehicle Symposium, pp. 564-569, Versailles, France, June 2002.

[2] W. Enkelmann, "Fleetnet- applications for inter-vehicle communication," in Proceedings of the IEEE Intelligent Vehicles Symposium, pp. 162-167, July 2003.

[3] P. Papadimitratos, A. La Fortelle, K. Evenssen, R. Brignolo, and S. Cosenza, "Vehicular communication systems: enabling technologies, applications, and future outlook on intelligent transportation," IEEE Communications Magazine, vol. 47, no. 11, pp. 84-95, 2009.

[4] C.-X. Wang, A. V. Vasilakos, R. D. Murch et al., "Guest Editorial: vehicular communications and networks-part I," IEEE Journal on Selected Areas in Communications, vol. 29, no. 1, pp. 1-6, 2011.

[5] W. Chen, Ed., Vehicular Communications and Networks: Architectures, Protocols, Operation and Deployment, Woodhead, Elsevier, 2015.

[6] A. F. Molisch, F. Tufvesson, J. Karedal, and C. F. Mecklenbräuker, "A survey on vehicle-to-vehicle propagation channels," IEEE Wireless Communications, vol. 16, no. 6, pp. 12-22, 2009.

[7] P. Belanović, D. Valerio, A. Paier, T. Zemen, F. Ricciato, and C. F. Mecklenbräuker, "On wireless links for vehicle-toinfrastructure communications," IEEE Transactions on Vehicular Technology, vol. 59, no. 1, pp. 269-282, 2010.

[8] C. Campolo and A. Molinaro, "On vehicle-to-roadside communications in 802.11p/WAVE VANETs," in Proceedings of the IEEE Wireless Communications and Networking Conference (WCNC '11), pp. 1010-1015, Cancun, Mexico, March 2011.

[9] L. Cheng, B. E. Henty, D. D. Stancil, F. Bai, and P. Mudalige, "Mobile vehicle-to-vehicle narrow-band channel measurement and characterization of the $5.9 \mathrm{GHz}$ dedicated short range communication (DSRC) frequency band," IEEE Journal on Selected Areas in Communications, vol. 25, no. 8, pp. 1501-1516, 2007.
[10] J. Maurer, T. Fügen, and W. Wiesbeck, "Narrow-band measurement analysis of the inter-vehicle transmission channel at $5.2 \mathrm{GHz}$," in Proceedings of the IEEE 55th Vehicular Technology Conference, vol. 3, pp. 1274-1278, Birmingham, Ala, USA, May 2002.

[11] I. Sen and D. W. Matolak, "Vehicle-vehicle channel models for the 5-GHz band," IEEE Transactions on Intelligent Transportation Systems, vol. 9, no. 2, pp. 235-245, 2008.

[12] H. Fernández, L. Rubio, V. M. Rodrigo-Peñarrocha, and J. Reig, "Path loss characterization for vehicular communications at 700 $\mathrm{MHz}$ and $5.9 \mathrm{GHz}$ under LOS and NLOS conditions," IEEE Antennas and Wireless Propagation Letters, vol. 13, pp. 931-934, 2014.

[13] O. Renaudin, V.-M. Kolmonen, P. Vainikainen, and C. Oestges, "Car-to-car channel models based on wideband MIMO measurements at $5.3 \mathrm{GHz}$," in Proceedings of the 3rd European Conference on Antennas and Propagation (EuCAP '09), pp. 635639, Berlin, Germany, March 2009.

[14] D. W. Matolak, R. Sun, and P. Liu, "V2V channel characteristics and models for $5 \mathrm{GHz}$ parking garage channels," in Proceedings of the 9th European Conference on Antennas and Propagation (EuCAP '15), Lisbon, Portugal, May 2015.

[15] L. Bernardó, A. Roma, A. Paier et al., "In-tunnel vehicular radio channel characterization," in Proceedings of the IEEE 73rd Vehicular Technology Conference (VTC '11), Budapest, Hungary, May 2011.

[16] V. Shivaldova, G. Maier, D. Smely, N. Czink, A. Paier, and C. F. Mecklenbräuker, "Performance analysis of vehicle-to-vehicle tunnel measurements at $5.9 \mathrm{GHz}$," in Proceedings of the 30th URSI General Assembly and Scientific Symposium (URSIGASS '11), IEEE, Istanbul, Turkey, August 2011.

[17] J.-M. Molina-Garcia-Pardo, M. Lienard, and P. Degauque, "Propagation in tunnels: experimental investigations and channel modeling in a wide frequency band for MIMO applications," EURASIP Journal on Wireless Communications and Networking, vol. 2009, Article ID 560571, pp. 1-9, 2009.

[18] H. Fernández, L. Rubio, J. Reig, V. M. Rodrigo-Peñarrocha, and A. Valero, "Path loss modeling for vehicular system performance and communication protocols evaluation," Mobile Networks and Applications, vol. 18, no. 6, pp. 755-764, 2013.

[19] L. Rubio, J. Reig, V. M. Rodrigo-Peñarrocha, H. Fernández, and S. Loredo, "Analysis of small-scale fading distributions in vehicular-to-vehicular communications," Mobile Information Systems, vol. 2016, Article ID 9584815, 7 pages, 2016.

[20] S. A. Abbas and A. U. Sheikh, "A geometric theory of Nakagami fading multipath mobile radio channel with physical interpretations," in Proceedings of the IEEE 46th Vehicular Technology Conference, Mobile Technology for the Human Race, Atlanta, Ga, USA, May 1996.

[21] F. Van der Wijk, A. Kegel, and R. Prasad, "Assessment of a pico-cellular system using propagation measurements at 1.9 $\mathrm{GHz}$ for indoor wireless communications," IEEE Transactions on Vehicular Technology, vol. 44, no. 1, pp. 155-162, 1995.

[22] F. J. Massey Jr., “The Kolmogorov-Smirnov test for goodness of fit," Journal of the American Statistical Association, vol. 46, no. 253, pp. 68-78, 1951.

[23] A. N. Kolmogorov, Selected Works, vol. II: Probability Theory and Mathematical Statistics, vol. 26, Kluwer Academic Publishers, Dordrecht, The Netherlands, 1992. 


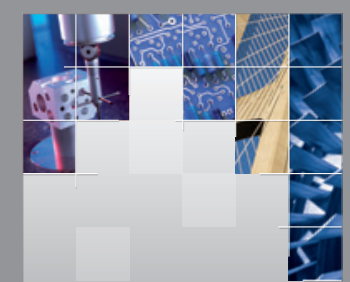

\section{Enfincering}
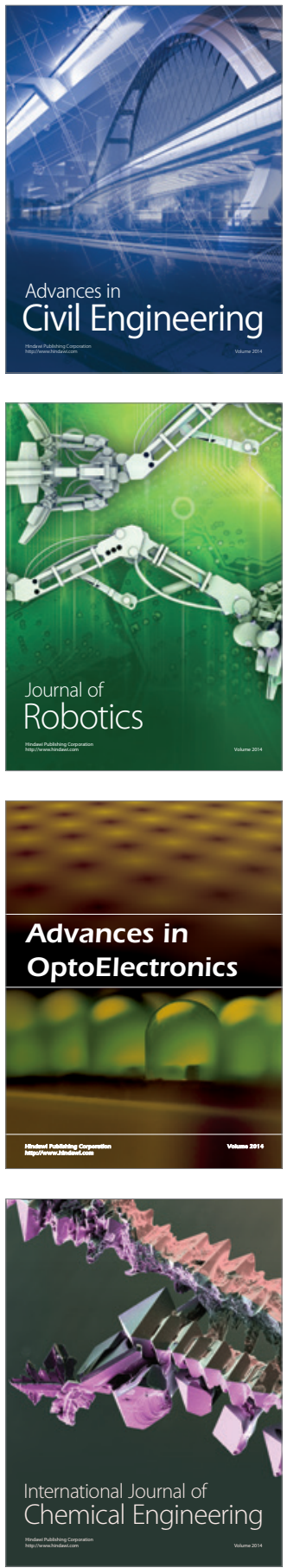

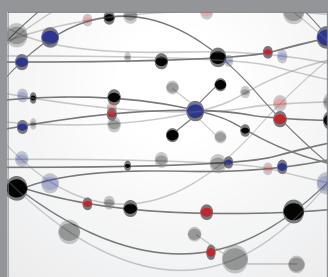

The Scientific World Journal

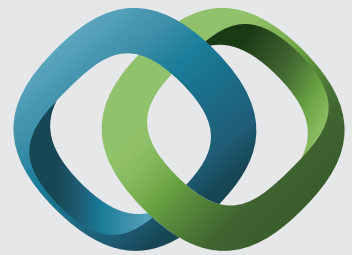

\section{Hindawi}

Submit your manuscripts at

https://www.hindawi.com
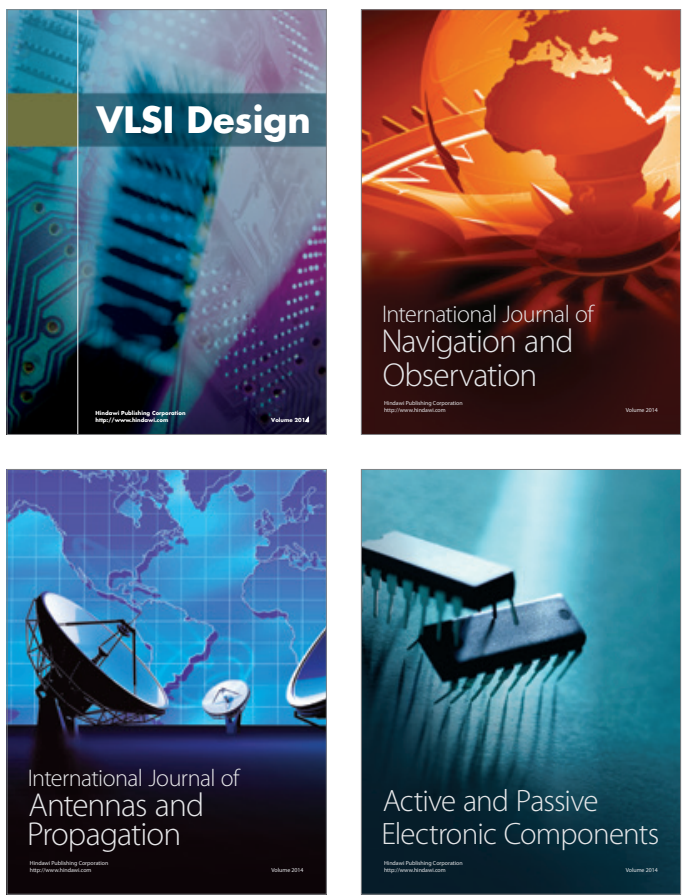
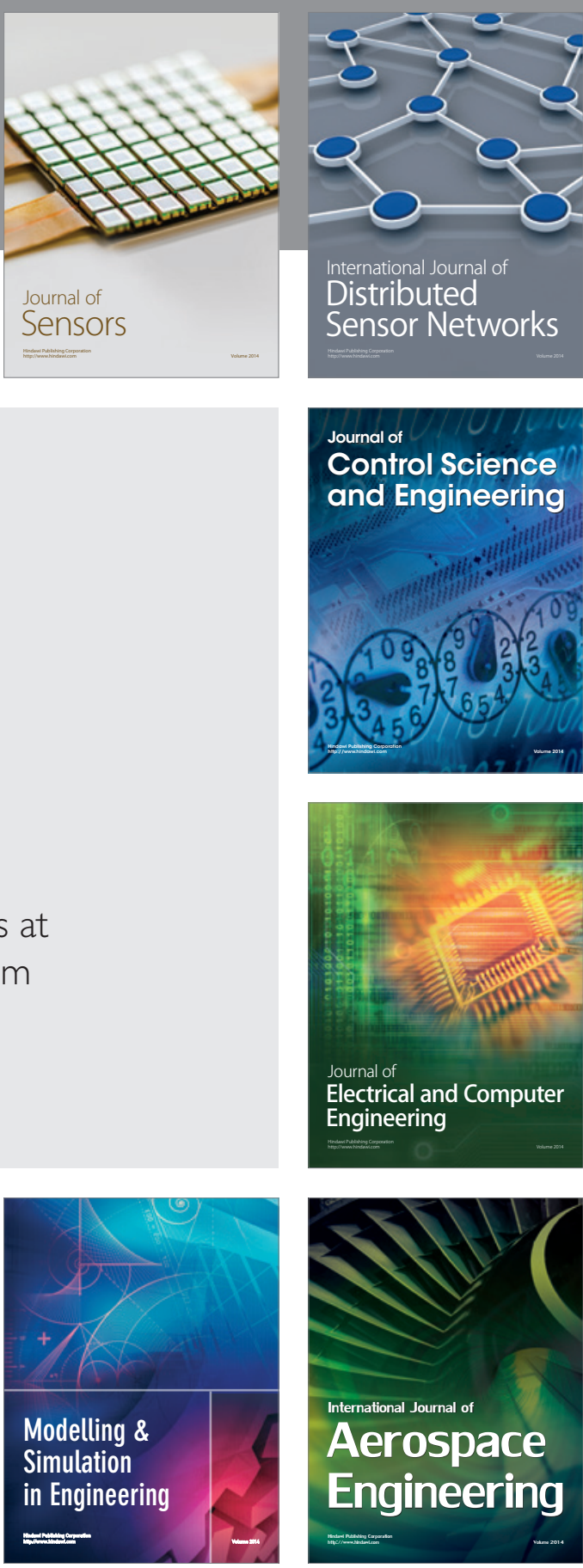

International Journal of

Distributed

Sensor Networks

$-$

Joumal of

Control Science

and Engineering
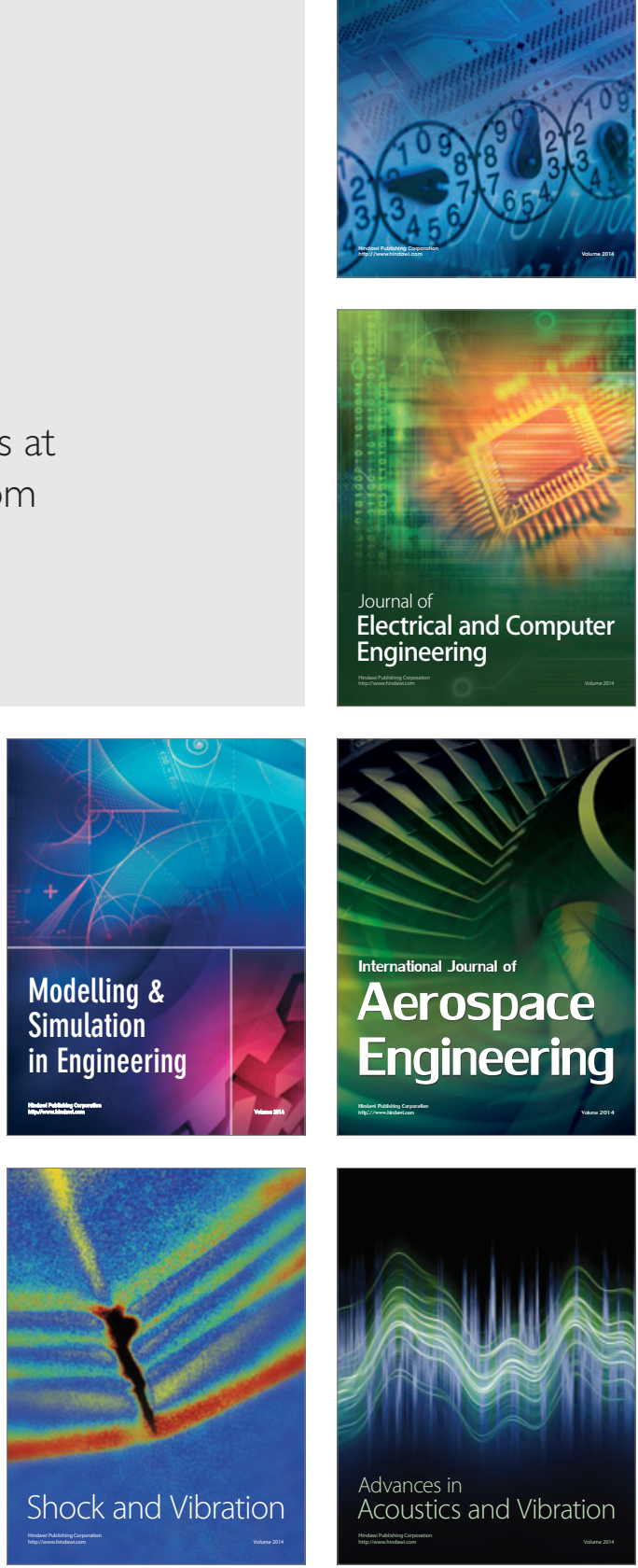